\title{
HT-KZ detector system: R\&D considerations and prototype performance
}

\author{
Tileubek Uakhitov ${ }^{1, \star}$, Ayan Batyrkhanov ${ }^{1}$, Dmitriy Beznosko ${ }^{3}$, Alexander lakovlev ${ }^{1}$, Shotan Jakupov, Amir \\ Kaipiyev ${ }^{4}$, Arailym Konysbayeva ${ }^{1}$, Alikhan Yeltokov ${ }^{1}$, Kamilya Yessimbet ${ }^{1}$, and Valeriy Zhukov ${ }^{2}$ \\ ${ }^{1}$ Cosmic Rays and Particles group,Nazarbayev University, Astana, $K Z$ \\ ${ }^{2}$ P. N. Lebedev Physical Institute of the Russian Academy of Sciences, Moscow, Russia \\ ${ }^{3}$ Bard Early College New Orleans, New Orleans, USA \\ ${ }^{4}$ NIS IB program, Astana, KZ
}

\begin{abstract}
.
Following the experience with the Horizon-10T detector systems, a completely new detector of Extensive Air Showers (EAS) has been designed to be built at Nazarbayev University, Astana, Kazakhstan. The motivation of building HorizonT-Kazakhstan is to study in detail the phenomenon of the multi-modal EAS events that were initially explored by Horizon-8T and 10T detector systems. This detector will have a ns-level resolution of charged particle arrival times and pulse shape resolution. Each detection point is designed to have a total scintillation area approximately equal to $7.7 \mathrm{~m}^{2}$ and consists of three $1.6 \times 1.6 \mathrm{~m}$ detectors located in a triangular arrangement for the local determination of the EAS disk arrival direction, and to have ns precision in timing between points. Local arrival direction is important following evidence of large angle differences in the multimodal events seen previously.
\end{abstract}

\section{Introduction}

HorizonT-Kazakhstan (HT-KZ) [1, 2] is a new EAS detector system that was designed based on experience gained from the Horizon-8T \& 10T experiments [3, 4] and intended to be build at Nazarbayev University, Astana, Kazakhstan. Detectors shown in the Figure 1 are prototypes for the scintillators and other equipment tests. Detection parameters of these prototype detectors such as rise time and light yield were tested and will be presented.

\section{Prototype detectors}

Prototype detectors were made using EJ-299-15 Cherenkov Radiator Plastic [5] as a base scintillation medium $(0.5 \times 0.5 \mathrm{~m})$ and photomultiplier tubes R7723 PMT Assembly from Hamamatsu [6]. The shape of detectors was designed according to simulation results [7]. Several designs were tested in simulations [8] and the pyramidal shape shown in Figure 2 was chosen because of sufficient light yield and uniformity of scintillated light propagation.

\subsection{Location description}

The prototype system was located at Nazarbayev University (NU) at $360 \mathrm{~m}$ height above sea level. According to our estimations at this height a range of EAS detection will start from $10^{16} \mathrm{eV}$ primary particle energy. In addition, prototypes were located on the balcony, with the building limiting a sky view, detectors relative position and detection angle.

\footnotetext{
^e-mail: tileubek.uakhitov@nu.edu.kz
}

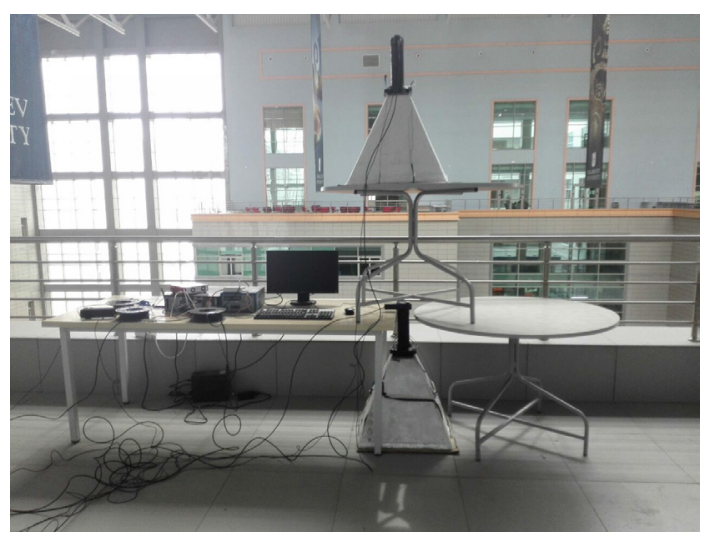

Figure 1. Prototype system under calibration

\subsection{Data acquisition and experimental procedure}

Data acquisition was done by using an analog to digital converter unit CAEN DT51730B [9] and a custom control software. The detection of an EAS was recorded when the signal from both detectors arrived in a window of $20 \mathrm{~ns}$.

During the experiment the distance between the prototype detectors was gradually increased and the rate of events arrival was recorded. The distance between the centers of detectors ranged from 0 to $19.2 \mathrm{~m}$. When the distance was $0 \mathrm{~m}$ one detector was placed on top of another and was a calibration stage. In the next stages both detectors were placed at the same height. For each step of the experiment 200 events were recorded and the time spent on each stage was noted. 


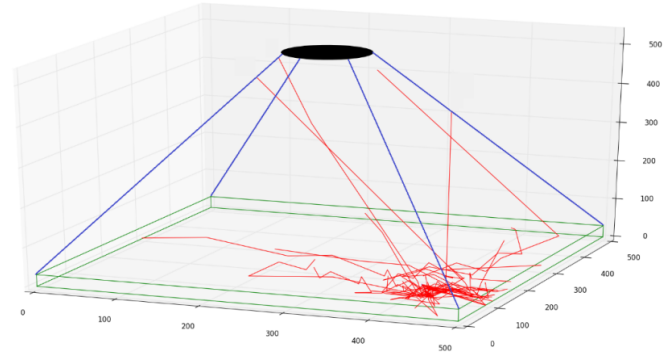

Figure 2. Simulation of particle detection in pyramid shape detector

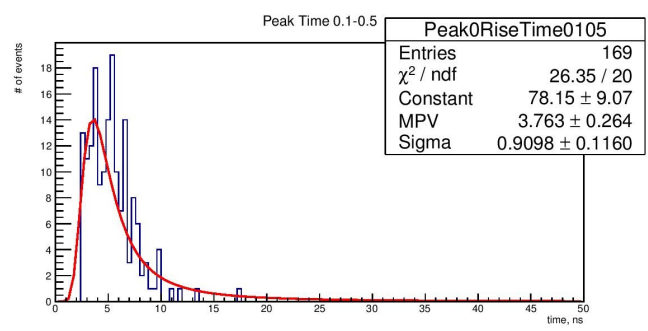

Figure 3. Rise time from 0.1 to 0.5 of pulse area

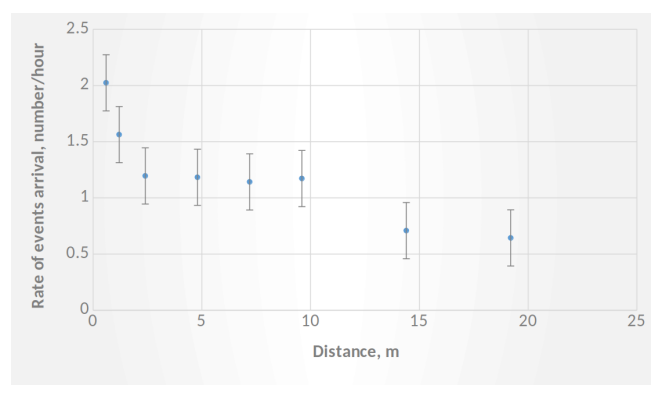

Figure 4. Rate of events vs detector separation

\section{Results of the experiment}

One of the important characteristics of the detector is the rise time. We define the rise time as the difference in time between $10 \%$ and $50 \%$ of the pulse area. In Figure 3 we can see that the rise time is $3.76 \pm 0.26$ ns which is more accurate than our prediction of $7 \mathrm{~ns}$.

According to experimental data the EJ-299-15 Cherenkov Radiator Plastic has insufficient light yield. The first calibration was done using one layer of $12.5 \mathrm{~mm}$ thin plastic. Next, the thickness of plastic was doubled but the increase in light yield was still insufficient. It resulted in problems with the detection of single minimally ionizing particle (MIP). Thus, to differentiate the signal from noise we used the calibration for several MIPs. Based on it we measured the rate of events arrival vs the distance between two detectors.
From Figure 4 it can be seen that from 1 to 10 meters the rate of events arrival is stable. This can be used in future for detector placement. Since the compromise between the detection rate and distance between them is needed to calculate the local angle accurately, the first trial will be done on the $10 \mathrm{~m}$ scale.

\section{Future Plans}

In continuation of this design of fast high energy particle detectors, we plan to place detectors in the form of an equilateral triangle. This configuration will give us an opportunity to measure the local angle of EAS. Each detector will have a base of $1.6 \times 1.6 \mathrm{~m}$ and will be placed at $10 \mathrm{~m}$ distance from the centers.

\section{Conclusion}

The first prototypes for HT-KZ detector system were constructed and tested. According to experimental results, it is necessary to replace the EJ-299-15 Cherenkov Radiator Plastic with another scintillation material for better light yield and to position detectors at $10 \mathrm{~m}$ distance from each other to calculate the local angle of EAS.

\section{Acknowledgments}

This work was partially funded by the MES RK statetargeted program BR95236464. Special thanks should be expressed to students of NIS high school: Sayan Imash, Ayan Kundybayev, Batyrkhan Mustafin and Artem Tsoy for participation in construction of the detectors.

\section{References}

[1] D Beznosko et al., arXiv:1803.08309 (2018)

[2] Rashid Beisembaev, et al., EPJ Web Conf. 14511004 (2017). DOI: 10.1051/epjconf/201614511004

[3] Rashid Beisembaev et al., EPJ Web Conf.145 14001 (2017). DOI: 10.1051/epjconf/201614514001 ISVHECRI

[4] R U Beisembaev et al., accepted to Journal of Physics: Conference series, (2018)

[5] Eljen Technology, 1300 W. Broadway, Sweetwater, Texas 79556, United States, www.eljentechnology.com

[6] Hamamatsu Photonics, 314-5 Shimokanzo, Toyooka-village, Iwatagun, Shizuoka-ken, 4380193 Japan http://www.hamamatsu.com

[7] R.U. Beisembaev, et al, JINST 12 (2017)

[8] A. Duspayev et al, arXiv:1703.07919 (2017)

[9] CAEN S.p.A. Via della Vetraia, 11, 55049 Viareggio Lucca, Italy http://www.caen.it 